\title{
OSCILLATION THEOREMS FOR SECOND-ORDER NONLINEAR DIFFERENTIAL EQUATIONS
}

\author{
JAMES S. W. WONG \\ (Communicated by Kenneth R. Meyer) \\ In memory of G. J. Butler
}

\begin{abstract}
Oscillation criteria for the second-order nonlinear differential equation $x^{\prime \prime}+a(t)|x|^{\gamma} \operatorname{sgn} x=0 \quad \gamma \neq 1$, are studied where the coefficient $a(t)$ is not assumed to be non-negative. New proofs are given to theorems of Butler, and extend earlier results of the author.
\end{abstract}

$\S 1$.

Consider the second-order scalar linear ordinary differential equation

$$
x^{\prime \prime}+a(t) x=0, \quad t \in[0, \infty),
$$

and $a(t) \in C[0, \infty)$. A solution of $(\mathrm{L})$ is said to be oscillatory it if has arbitrarily large zeros, i.e. for any $t_{0} \in[0, \infty)$, there exists $t_{1} \geq t_{0}$ such that $x(t+1)=0$. Equation (L) is said to be oscillatory if all solutions are oscillatory. The most important simple oscillation criterion is the well-known Fite-Wintner theorem which states that if $a(t)$ satisfies

$$
\lim _{T \rightarrow \infty} A(T)=\lim _{T \rightarrow \infty} \int_{0}^{T} a(t) d t=+\infty,
$$

then equation $(\mathrm{L})$ is oscillatory. Fite [7] assumed in addition that $a(t)$ is nonnegative, whilst Wintner [15] in fact proved a stronger result which required a weaker condition involving the integral average of $A(t)$;

$$
\lim _{T \rightarrow \infty} \frac{1}{T} \int_{0}^{T} A(t) d t=+\infty .
$$

Condition $\left(\mathrm{H}_{0}\right)$ was discovered independently by Leighton [12] so it is also referred in some earlier literature as the Fite-Wintner-Leighton oscillation theorem. Wintner's result was substantially improved by Hartman [8] who proved that $\left(\mathrm{H}_{1}\right)$ can be replaced by two weaker conditions

Received by the editors November 16, 1987 and, in revised form March 28, 1988.

1980 Mathematics Subject Classification (1985 Revision). Primary 34C10, 34C15.

Key words and phrases. Second order, nonlinear, ordinary differential equations, oscillation, asymptotic behavior. 


$$
\liminf _{T \rightarrow \infty} \frac{1}{T} \int_{0}^{T} A(t) d t>-\infty,
$$

and that the limit in $\left(\mathrm{H}_{1}\right)$ does not exist, i.e.

$$
\lim _{T \rightarrow \infty} \frac{1}{T} \int_{0}^{T} A(t) d t<\varlimsup_{T \rightarrow \infty} \frac{1}{T} \int_{0}^{T} A(t) d t .
$$

In a different direction, Kamenev [11] showed that if for some positive integer $n>1$,

$$
\lim _{T \rightarrow \infty} \sup \frac{1}{T} \int_{0}^{T}(T-t)^{n} a(t) d t=+\infty
$$

then $(\mathrm{L})$ is oscillatory. It is also known that the condition $\left(\mathrm{H}_{4}\right)$ with $n=1$, which may be rewritten as

$$
\limsup _{T \rightarrow \infty} \frac{1}{T} \int_{0}^{T} A(t) d t=+\infty
$$

alone is not sufficient for the oscillation of (L), see Hartman [9], Willett [14].

A natural generalization of equation $(\mathrm{L})$ is to the following second-order nonlinear differential equation

$$
x^{\prime \prime}+a(t)|x|^{\gamma} \operatorname{sgn} x=0, \quad t \in[0, \infty),
$$

where $a(t) \in C[0, \infty), \gamma>0$ and $\operatorname{sgn} x(t)$ denotes the sign of the solution $x(t)$. The special case when $a(t)=t^{\sigma}$ is the so called Emden-Fowler equation which arises originally from the study of astrophysics. We refer the interested reader to the survey article [19] on equation $\left(\mathbf{N}_{\gamma}\right)$ and its many interesting results when $a(t)$ is in addition non-negative, a condition which was not assumed throughout this paper. We are here concerned with the extension of oscillation criteria $\left(\mathbf{H}_{0}\right)-\left(\mathbf{H}_{5}\right)$ for equation $(\mathrm{L})$ to the nonlinear equation $\left(\mathbf{N}_{\gamma}\right), \gamma>0$.

First of all, we wish to point out that not every solution of $\left(\mathbf{N}_{\gamma}\right)$ is continuable throughout $[0 \infty)$ when $a(t)$ can assume negative values for arbitrarily large value of $t$. Thus, we say equation $\left(N_{\gamma}\right)$ is oscillatory if every continuable solution is oscillatory. For results on continuability of solutions of $\left(\mathbf{N}_{\gamma}\right)$, we refer to [19], and Butler [4]. We list below the major results relating to the generalization criteria $\left(\mathrm{H}_{0}\right)-\left(\mathrm{H}_{5}\right)$, that of Wintner, Hartman and Kamenev, to equation $\left(\mathbf{N}_{\gamma}\right)$ :
(I) Waltman [13],
$\left(\mathbf{H}_{0}\right) \Rightarrow\left(\mathbf{N}_{\gamma}\right)$ oscillatory, $\quad \gamma>0$.
(II) Kamenev [10],
$\left(\mathbf{H}_{5}\right) \Rightarrow\left(\mathbf{N}_{\gamma}\right)$ oscillatory, $0<\gamma<1$.
(III) Butler
[5],
$\left(\mathbf{H}_{1}\right) \Rightarrow\left(\mathbf{N}_{\gamma}\right)$ oscillatory, $\quad \gamma>0$.
(IV) Butler
[5],
$\left(\mathbf{H}_{2}\right)\left(\mathbf{H}_{2}\right) \Rightarrow\left(\mathbf{N}_{\gamma}\right)$ oscillatory, $\quad \gamma>1$.
(V) Butler
[5],
$\left(\mathbf{H}_{3}\right) \Rightarrow\left(\mathbf{N}_{\gamma}\right)$ oscillatory, $0<\gamma<1$.

Kamenev's criterion $\left(\mathrm{H}_{4}\right)$ for the linear equation $(\mathrm{L})$ has recently been shown together with the condition

$$
\liminf _{T \rightarrow \infty} \int_{0}^{T} a(t) d t=-\lambda>-\infty
$$


to yield

(VI) Wong [20], $\left(\mathbf{H}_{4}\right),\left(\mathrm{H}_{6}\right) \Rightarrow\left(\mathrm{N}_{\gamma}\right)$ oscillatory, $\gamma>0$.

Condition $\left(\mathrm{H}_{6}\right)$ was earlier used to prove

(VI) Wong [17], $\left(\mathrm{H}_{t}\right),\left(\mathrm{H}_{6}\right) \Rightarrow\left(\mathrm{N}_{\gamma}\right)$ oscillatory, $\gamma>1$.

With the exception that (III) $\Rightarrow$ (I) since condition $\left(\mathrm{H}_{0}\right)$ follows from $\left(\mathrm{H}_{1}\right)$, all other six oscillation theorems are independent of one another. Amongst these, the most sophisticated are the three theorems of Butler (III), (IV), and (V) which were in fact proved for the more general equation

$$
x^{\prime \prime}+a(t) f(x)=0, \quad t \in[0, \infty),
$$

where $f(x 0 \in C(-\infty, \infty)$ and $x f(x)>0$ when $x \neq 0$, together with some rather complicated assumptions which are satisfied for $f(x)=|x|^{\gamma} \operatorname{sgn} x, \gamma>$ 0 . In short, Butler showed that results of Wintner and Hartman for the linear equation $(\mathrm{L})$ remained valid for the nonlinear equation $\left(\mathrm{N}_{\gamma}\right)$, the desired extension resolving an open problem for some years.

Butler's proofs for these results are unfortunately rather technical, therefore the purpose of this paper is to give substantially simpler proofs, making use of the more special form of equation $\left(\mathrm{N}_{\gamma}\right)$. This approach can also be used to improve our earlier results (VI) and (VII) as follows:

$$
\begin{array}{ll}
(\mathrm{VI})^{\prime} & \left(\mathrm{H}_{2}\right),\left(\mathrm{H}_{4}\right) \Rightarrow\left(\mathrm{N}_{\gamma}\right) \text { oscillatory, } \gamma>0 . \\
(\mathrm{VII})^{\prime} & \left(\mathrm{H}_{3}\right),\left(\mathrm{H}_{6}\right) \Rightarrow\left(\mathrm{N}_{\gamma}\right) \text { oscillatory, } \gamma>1 .
\end{array}
$$

$\S 2$.

To prove that $\left(\mathrm{N}_{\gamma}\right)$ is oscillatory, we assume the existence of a positive solution $x(t)$ on $\left[t_{0}, \infty\right)$, and deduce a contradiction from conditions $\left(H_{1}\right)$, $\left(\mathrm{H}_{2}\right),\left(\mathrm{H}_{3}\right),\left(\mathrm{H}_{4}\right)$, and $\left(\mathrm{H}_{t}\right)$, as case may be, to prove (III), (IV), (V), (VI)', $(\mathrm{VIII})^{\prime}$. Define $y(t)=x^{1-\gamma}(t)$ when $\gamma \neq 1$. It is easy to verify from $\left(\mathrm{N}_{\gamma}\right)$ that $y(t)$ satisfies the second-order nonlinear differential equation

$$
y^{\prime \prime}-(\gamma-1) a+\gamma(\gamma-1)^{-12} y^{-1} y^{\prime 2},
$$

on $\left[t_{0}, \infty\right)$. Denote $\alpha=\gamma-1$ and $\beta=\gamma(\gamma-1)^{-1}$. We also define $\sigma(t)=$ $y^{\prime}(t)-\alpha A(t)$, where $A(t)=\int_{0}^{t} a(s) d s$. Integrating (1) from $t_{0}$ to $t$, we obtain

$$
\sigma(t)=\sigma\left(t_{0}\right)+\beta \int_{t_{0}}^{t} y^{-1} y^{\prime 2} \text {. }
$$

Note that if $\gamma>1$ then $\alpha>0, \beta>1$ and if $0<\gamma<1$ then $-1<\alpha<0$, $\beta<-1$. Integrating (2) one again and dividing by $t$, we have

$$
\frac{-\beta}{t} \int_{t_{0}}^{t} \int_{t_{0}}^{s} y^{-1} y^{\prime 2}+\frac{1}{t} y(t)=\sigma\left(t_{0}\right)+\frac{1}{t} y\left(t_{0}\right)+\alpha t \int_{t_{0}}^{t} A \text {. }
$$


We distinguish two mutually exclusive cases, (i) $y^{-1} y^{\prime 2} \in L^{1}\left(t_{0}, \infty\right)$ and (ii) $y^{-1} y^{\prime 2} \notin L^{1}\left(t_{0}, \infty\right)$ and verify that oscillation criteria given in (III), (IV), (V), $(\mathrm{VI})^{\prime}$, and (VII) ${ }^{\prime}$ would in each case lead to a desired contradiction. In the first case when $y^{-1} y^{\prime 2} \in L^{1}\left(t_{0}, \infty\right)$, we shall prove that

$$
\lim _{t \rightarrow \infty} \frac{1}{t} y(t)=0
$$

Let $\varepsilon>0$, choose $t_{1} \geq t_{0}$ such that $\int_{t_{1}}^{\infty} y^{-1} y^{\prime 2}<\varepsilon / 4$. Using Schwarz's Inequality, we can estimate as follows:

$$
y(t)-y\left(t_{1}\right)=\int_{t_{1}}^{t} y^{\prime} \leq \sqrt{\int_{t_{1}}^{t} y^{-1} y^{\prime 2}} \sqrt{\int_{t_{1}}^{t} y} \leq \frac{\sqrt{\varepsilon}}{2} \sqrt{\int_{t_{1}}^{t} y} .
$$

Suppose that $y \in L^{1}\left(t_{1}, \infty\right)$, then (5) shows that $y(t)$ is bounded, hence (4) follows. Assume that $y \notin L^{1}(t, \infty)$ so one can choose $t_{2} \geq t_{1}$ so that $y^{2}\left(t_{1} 0 \leq\right.$ $\varepsilon / 4 \int_{t_{1}}^{t} y$ for $t \geq t_{2}$. Using this in (5), we note that for $t \geq t_{2}$,

$$
y(t) \leq y\left(t_{1}\right)+\frac{\sqrt{\varepsilon}}{2} \sqrt{\int_{t_{1}}^{t} y} \leq\left(\frac{\sqrt{\varepsilon}}{2}+\frac{\sqrt{\varepsilon}}{2}\right) \sqrt{\int_{t_{1}}^{t} y} .
$$

Dividing (6) through by $\sqrt{\int_{t_{1}}^{t} y}$ and integrating from $t_{2}$ to $t$, we obtain

$$
\sqrt{\int_{t_{1}}^{t} y}-\sqrt{\int_{t_{1}}^{t_{2}} y} \leq \frac{\sqrt{\varepsilon}}{2}\left(t-t_{2}\right) \leq \frac{\sqrt{\varepsilon}}{2} t
$$

Once again we can choose $t_{3} \geq t_{2}$ so that $\int_{t_{1}}^{t_{2}} y \leq \varepsilon t^{2} / 4$ for $t \geq t_{3}$ which upon substituting in (7) and then (6) yield $y(t) \leq \varepsilon t$ for $t \geq t_{3}$. This proves assertion (4).

Returning to (3) and denoting $B_{0}=\int_{t_{0}}^{\infty} y^{-1} y^{\prime 2}$, we can pass the limit $t$ to infinity and find

$$
\lim _{t \rightarrow \infty} \frac{1}{t} \int_{0}^{t} A(s) d s=\frac{-\beta}{\alpha} B_{0}<0
$$

Clearly, (8) is incompatible with $\left(\mathrm{H}_{1}\right),\left(\mathrm{H}_{3}\right)$, hence contradiction results for (III), (IV), (V), and (VII) ${ }^{\prime}$. In so far as (VI) ${ }^{\prime}$ is concerned, the proof given in [20], in this case when $y^{-1} y^{\prime 2} \in L^{1}\left(t_{0}, \infty\right)$, remains valid because condition $\left(\mathrm{H}_{6}\right)$ was never used in the proof. 
We now turn to the more difficult case when $y^{-1} y^{\prime 2} \notin L^{1}\left(t_{0}, \infty\right)$. First, consider $\gamma>1$, then $\beta>1$ and we can choose $\mu, \mu_{1}$ such that $1<\mu<\mu_{1}<$ $\beta$. Claim

$$
\limsup _{t \rightarrow \infty}\left(\mu_{1} \int_{t_{0}}^{t} \int_{t_{0}}^{s} y^{-1} y^{\prime 2}-y(t)\right)>0
$$

Denote the double integral in (9) by $\Phi(t)$ and assume that (9(does not hold, then there exists $t_{1}$ such that

$$
\Phi(t) \leq\left(1 / \mu_{1}\right) y(t), \quad \text { for } t \geq t_{1} .
$$

It is easy to see that $\Phi(t) \rightarrow \infty, \Phi^{\prime}(t) \rightarrow \infty$, as $t \rightarrow \infty$, and $\Phi^{\prime \prime}(t)=$ $y^{-1}(t) y^{\prime 2}(t)$. Using (10), we have the following estimate

$$
\sqrt{\frac{\Phi^{\prime \prime}(t)}{\Phi^{\prime}(t)}} \sqrt{\frac{\Phi^{\prime}(t)}{\Phi(t)}}=\frac{y^{\prime}(t)}{\sqrt{y(t) \Phi(t)}} \geq \sqrt{\mu_{1}} \frac{y^{\prime}(t)}{y(t)} \text {. }
$$

Note that $\Phi\left(t_{0}\right)=0$, so we may choose $\Phi\left(t_{2}\right)=1$. Now integrating (11) from $t_{2}$ to $t$ and applying Schwarz's inequality to the left-hand side of (11), we obtain

$$
\sqrt{\log \frac{\Phi^{\prime}(t)}{\Phi^{\prime}\left(t_{2}\right)}} \sqrt{\log \Phi(t)} \geq \sqrt{\mu_{1}} \log \frac{y(t)}{y\left(t_{2}\right)} .
$$

Using (10), we can estimate the right-hand side of (12) as follows:

$$
\sqrt{\mu_{1}} \log \frac{y(t)}{y\left(t_{2}\right)}=\sqrt{\mu_{1}}\left(\log \Phi(t)+\log \mu_{1}-\log y\left(t_{2}\right)\right) .
$$

Since $\Phi(t) \rightarrow \infty$ as $t \rightarrow \infty$ and $\mu_{1}>\mu$, the right-hand side of (13) is bounded below by $\sqrt{\mu} \log \Phi(t)$, which can then be used to simplify (12) as follows

$$
\log \frac{\Phi^{\prime}(t)}{\Phi^{\prime}\left(t_{2}\right)} \geq \mu \log \Phi(t),
$$

or

$$
\Phi^{-\mu}(t) \Phi^{\prime}(t) \geq \Phi^{\prime}\left(t_{2}\right)>0 .
$$

Because $\mu>1$, integrating (14) and passing the limit would produce the desired contradiction. Thus assertion (9) holds and there exists a sequence $\left\{t_{k}\right\}$ such that

$$
\lim _{k \rightarrow \infty}-\mu_{1} \Phi\left(t_{k}\right)+y\left(t_{k}\right)<0 .
$$

Using (15) in (3), we find for sufficiently large $t_{k}$,

$$
\left(-\beta+\mu_{1}\right) \frac{1}{t_{k}} \Phi\left(t_{k}\right) \geq \sigma\left(t_{0}\right)+\frac{1}{t_{k}} y\left(t_{0}\right)+\frac{\alpha}{t_{k}} \int_{t_{0}}^{t_{k}} A .
$$

Since $\mu_{1}<\beta$ and $(1 / t) \Phi(t) \rightarrow \infty$ as $t \rightarrow \infty$ (16) and $\alpha>0$ imply 


$$
\liminf _{t \rightarrow \infty} \frac{1}{t} \int_{0}^{t} A(s) d s=-\infty
$$

which is a contradiction to $\left(\mathrm{H}_{2}\right)$. This proves (IV), and (VI) ${ }^{\prime}$.

Note that (17) is also incompatible with $\left(\mathrm{H}_{1}\right)$, hence (III) follows when $\gamma>1$. Similarly, (17) is also incompatible with $\left(\mathrm{H}_{2}\right)$, so $(\mathrm{VI})^{\prime}$ is proved for $\gamma>1$.

Finally, we need to prove (V) and (III), (VI) ${ }^{\prime}$ when $0<\gamma<1$. In this case, $\alpha<0, \beta<-1$. The left-hand side of (3) tends to infinity as $t \rightarrow \infty$, hence (17) must follow, and (V) is proved. Once again (17) is also incompatible with $\left(\mathrm{H}_{2}\right)$ and $\left(\mathrm{H}_{2}\right)$, proving (III), $(\mathrm{VI})^{\prime}$ for $0<\gamma<1$. The proof of all assertions is now complete.

\section{$\S 3$.}

In this last section, we assume in addition that $\lim _{t \rightarrow \infty} A(t)$ exists as a finite number, best known as the integrable coefficient case. For results the linear equation (L), we refer to Wong [16]. Denote $\bar{A}(t)=\int_{t}^{\infty} a(s) d s$ and $\bar{A}_{+}(t)=$ $\max (A(t), 0)$. The following conditions are considered in Coles [6] and Butler [3].

$$
\begin{gathered}
\lim _{T \rightarrow \infty} \int_{0}^{T} \bar{A}(t) d t=+\infty, \\
\liminf _{T \rightarrow \infty} \int_{0}^{T} \bar{A}(t) d t>-\infty,
\end{gathered}
$$

and

$$
\lim _{T \rightarrow \infty} \int_{0}^{T} \int_{t}^{\infty} \bar{A}_{+}(t) d t=+\infty
$$

As a further extension of a well-known result of Atkinson [1], Coles [6] proved

$$
\text { (VIII) Butler [3] } \quad\left(\mathrm{H}_{7}\right) \Rightarrow\left(\mathrm{N}_{\gamma}\right) \text { oscillatory, } \gamma>1 .
$$

under the additional assumption that $\bar{A} 9 t) \geq 0$. In the same paper, Butler also proved

$$
\text { (IX) Butler [3] }\left(H_{8}\right),\left(\mathbf{H}_{9}\right) \Rightarrow\left(\mathbf{N}_{\gamma}\right) \text { oscillatory, } \gamma>1 .
$$

Once again, these are best results on $\left(\mathrm{N}_{\gamma}\right), \gamma>1$, known to date when $a(t)$ is integrable. Note that neither condition $\left(\mathrm{H}_{7}\right)$ nor $\left(\mathrm{H}_{8}\right)$ is sufficient for oscillation of $(\mathrm{L})$, as $a(t)=\frac{1}{4} t^{-2}$ attests. In the sublinear case, we can have the corresponding

$$
\begin{aligned}
& \left(\mathrm{H}_{7}\right) \Rightarrow \text { Every bounded solution of }\left(\mathrm{N}_{\gamma}\right) \text {, } \\
& 0<\gamma<1 \text {, is oscillatory. } \\
& \left(\mathrm{H}_{9}\right) \Rightarrow \text { Every bounded solution of }\left(\mathrm{N}_{\gamma}\right) \text {, } \\
& 0<\gamma<1 \text {, is oscillatory. }
\end{aligned}
$$


Note that the boundedness requirement in $(\mathrm{X})$ and $(\mathrm{XI})$ cannot be removed, because when $a(t)=t^{-1}$, equation $\left(\mathrm{N}_{\gamma}\right), 0<\gamma<1$, has unbounded nonoscillatory solutions, consequence of another well-known result due to Belohorec [2]. Results weaker than (VIII) and $(\mathrm{X})$ involving condition $\left(\mathrm{H}_{7}\right)$ may also be found in [18].

We shall show in this section that techniques developed in the preceding section can also be used to provide considerably simply proofs to these results.

We first return to equation (1) and integrate from $t$ to $T$

$$
y^{\prime}(T)-y^{\prime}(t)=\alpha \int_{t}^{T} a(s) d s+\beta \int_{t}^{T} y^{-1} y^{\prime 2} .
$$

Suppose that $y^{-1} y^{\prime 2} \in L^{1}\left(t_{0}, \infty\right)$. From (18) we deduce that $\lim _{t \rightarrow \infty} y^{\prime}(t)=m$. Claim $m=0$. If not and suppose that $m<0$, then $y(t) \rightarrow-\infty$ as $t \rightarrow \infty$ contradicting that $y(t)>0$. Let $m>0$, then for large $t$ we have

$$
y^{\prime}(t) \geq m / 2 \text { and } y(t) \leq 2 m t,
$$

which together contradict $y^{-1} y^{\prime 2} \in L^{1}\left(t_{0}, \infty\right)$. Now pass the limit in (18) as $T \rightarrow \infty$, we obtain

$$
y^{\prime}(t)+\alpha \bar{A}(t)+\beta \int_{t}^{\infty} y^{-1} y^{\prime 2}=0 .
$$

We show that (20) remains valid even in the case $y^{-1} y^{\prime 2} \notin L^{1}\left(t_{0}, \infty\right)$. To see this, first consider $\gamma>1$, so $\beta>1$, and one can choose $\mu, 1<\mu<\beta$. Since the last integral becomes arbitrarily large as $T \rightarrow \infty$, we can estimate (18) by choosing $t_{1}$ sufficiently large so that for $T \geq t_{1}>t_{0}$,

$$
y^{\prime}(t) \geq \mu \int_{t}^{T} y^{-1} y^{\prime 2}=\mu R(T) .
$$

Rewriting $T$ and $t$ in (21) by $t$ and $t_{0}$, we note $y^{\prime}(t) \geq \mu R(t)$ when $t \geq t_{1}$. Multiplying (21) by $y^{-1} y^{\prime}$ on both sides, we obtain

$$
R^{\prime}(t)=y^{-1} y^{\prime 2}(t) \geq \mu R(t) y^{-1} y^{\prime}(t) .
$$

Dividing (22) through by $R(t)$ and integrating from $t_{1}$ to $t$, we have

$$
\log \frac{R(t)}{R\left(t_{1}\right)} \geq \mu \log \frac{y(t)}{y\left(t_{1}\right)},
$$

from which together with (21) one obtains

$$
y^{\prime}(t) \geq \mu R(t) \geq \mu R\left(t_{1}\right)\left(\frac{y(t)}{y\left(t_{1}\right)}\right)^{\mu} .
$$

Since $\mu>1$, another quadrature of (23) yields a contradiction. In the sublinear case $0<\gamma<1$, we have $\beta<0$. Turning to (18) once again, it follows that $\lim _{t \rightarrow \infty} y^{\prime}(t)=-\infty$ which is incompatible with $y(t)>0$. Hence $\left.y^{-1} y^{\prime 2} \notin L^{1} 9 t_{0}, \infty\right)$ cannot happen when $\gamma \neq 1$. Therefore, equation (20) holds whenever $A(t)$ has a finite limit as $t \rightarrow \infty$. 
To prove (VIII), we return to (20) and drop the integral term to obtain

$$
y^{\prime}(t) \leq-\alpha \bar{A}(t),
$$

which upon integrating and applying $\left(\mathrm{H}_{7}\right)$ yield $\lim _{t \rightarrow \infty} y(t)=-\infty$. This is a desired contradiction, proving (VIII). To prove (IX), we note from (24) that for $t \geq t_{1}$, one has

$$
y^{\prime 2}(t) \geq \alpha^{2} \bar{A}_{+}^{2}(t) .
$$

Suppose that $\left(\mathrm{H}_{7}\right)$ fails (for otherwise (VIII) is applicable hence oscillation results), then $\left(\mathrm{H}_{8}\right)$ implies that $\left|\int_{t_{1}}^{t} \bar{A}(s) d s\right| \leq M$, where $M$ may depend on $t_{1}$. Integrating (24) we find

$$
0<y(t) \leq y\left(t_{1}\right)+\alpha\left|\int_{t_{1}}^{t} \bar{A}(s) d s\right| \leq M_{1},
$$

where $M_{1}=y\left(t_{1}\right)+\alpha M$. Returning to (20) and integrating, one obtains

$$
y(t) \leq y\left(t_{0}\right)+\alpha|| \int_{t_{1}}^{t} \bar{A}(s) d s \mid-\beta \int_{t_{1}}^{t} \int_{s}^{\infty} y^{-1} y^{\prime 2} .
$$

Using (25) and (26), we can estimate the last integral in (27) as follows:

$$
\int_{t_{1}}^{t} \int_{s}^{\infty} y^{-1} y^{\prime 2} \geq M_{1}^{-1} \int_{t_{1}}^{t} \int_{s}^{\infty} \bar{A}_{+}^{2}(u) d u,
$$

which tends to infinity as $t \rightarrow \infty$ by $\left(\mathrm{H}_{9}\right)$. This together with (27) imply that $y(t) \rightarrow-\infty$, a desired contradiction, proving (IX).

For the sublinear case (X), we note that $\alpha<0, \beta<-1$, so the effect of dropping the integral term in (20) gives

$$
y^{\prime}(t) \geq-\alpha \bar{A}(t) .
$$

Again integrating (29), one deduces that if $y(t)$ is nonoscillatory, then $y(t) \rightarrow \infty$ as $t \rightarrow \infty$. Hence, every bounded solution of $\left(\mathrm{N}_{\gamma}\right), 0<\gamma<1$, must be oscillatory. Since $\alpha<0,(29)$ implies that (25) also holds. Using the fact that $y(t)$ is bounded and repeating the argument in (27) and (28), one easily obtains the desired contradiction. The proof of $(\mathrm{XI})$ is complete.

\section{REFERENCES}

1. F. V. Atkinson, On second nonlinear oscillation, Pacific J. Math. 5 (1955), 643-647.

2. S. Belohorec, Oscillatory solutions of certain nonlinear differential equations of the second order, Mat. Casopis Sloven. Akad. Vied. 11 (1961), 250-255.

3. G. J. Butler, On the oscillatory behaviour of a second order nonlinear differential equation, Ann. Mat. Pura Appl. 105 (1975), 73-92.

4. G. J. Butler, The existence of continuable solutions of a second order differential equation, Canad. J. Math. 29 (1977), 472-479.

5. G. J. Butler, Integral averages and the oscillation of second order ordinary differential equations, SIAM J. Math. Anal. 11 (1980), 190-200. 
6. W. J. Coles, Oscillation criteria for nonlinear second order equations, Ann. Mat. Pura Appl. 82 (1969), 123-134.

7. W. B. Fite, Concerning the zeros of the solutions of certain differential equations, Trans. Amer. Math. Soc. 19 (1918), 341-352.

8. P. Hartman, On nonoscillatory linear differential equations of second order, Amer. J. Math. 74 (1952), 389-400.

9. P. Hartman, Ordinary differential equations, Wiley, New York, 1964.

10. I. V. Kamenev, Some specifically nonlinear oscillation theorems, Mat. Zametki 10 (1971), 129-134.

11. I. V. Kamenev, Integral criterion for oscillations of linear differential equations of second order, Mat. Zametki 23 (1978), 249-251.

12. W. Leighton, The detection of the oscillation of solutions of a second order linear differential equation, Duke Math. J. 17 (1950), 57-62.

13. P. Waltman, An oscillation criterion for a nonlinear second order equation, J. Math. Anal. Appl. 10 (1965), 439-441.

14. D. Willett, On the oscillatory behavior of the solutions of second order linear differential equations, Ann. Polon. Math. 21 (1969), 175-194.

15. A. Wintner, A criterion of oscillatory stability, Quart. J. Appl. Math. 7 (1949), 115-119.

16. J. S. W. Wong, Oscillation and nonoscillation of solutions of second order linear differential equations with integrable coefficients, Trans. Amer. Math. Soc. 144 (1969), 197-215.

17. __ A second order nonlinear oscillation theorem, Proc. Amer. Math. Soc. 40 (1973), 487491.

18. __ Oscillation theorems for second order nonlinear differential equations, Bull. Inst. Math. Acad. Sinica 3 (1975), 283-309.

19. __, On the generalized Emden-Fowler equation, SIAM Rev. 17 (1975), 339-360.

20. _ An oscillation criterion for second order nonlinear differential equations, Proc. Amer. Math. Soc. 98 (1986), 109-112.

Chinney Investments Ltd., 814 Swire Houre, Hong Kong

University of Hong Kong, Pokfulam, Hong Kong 